\title{
A randomised trial of Supine versus Prone breast radiotherapy (SuPr study): comparing set-up errors and respiratory motion
}

\author{
Kirby $\mathrm{AM}^{1}$, Evans $\mathrm{PM}^{2}$, Helyer $\mathrm{S}^{1}$, Donovan $\mathrm{EM}^{1}$, Convery $\mathrm{HM}^{1}$, Yarnold $\mathrm{JR}^{2}$ \\ ${ }^{1}$ Royal Marsden NHS Foundation Trust, Sutton, UK and ${ }^{2}$ Institute of Cancer Research, Sutton, UK
}

Corresponding author: Dr Anna M. Kirby FRCR, Department of Academic Radiotherapy, Royal Marsden NHS Foundation Trust, Downs Road, Sutton, Surrey, SM2 5PT, United Kingdom. Telephone: +44 208661 3169. Fax: +44 208661 3107. E-mail: anna.kirby@rmh.nhs.uk

Acknowledgements: $\operatorname{Dr}$ A Kirby was funded by a Royal College of Radiologists' Clinical Oncology Research Fellowship and by the Breast Cancer Research Trust. The authors are grateful to Silvia Formenti and colleagues at New York University Hospital for their advice on the prone breast platform and technique, and to Mr Craig Cummings in the Institute of Cancer Research Workshop for his assistance in designing and manufacturing our prone breast platform. The work was undertaken in The Royal Marsden NHS Foundation Trust which receives a proportion of its funding from the NHS Executive; the views expressed in this publication are those of the authors and not necessarily those of the NHS Executive.

Key words: Breast cancer; Prone breast radiotherapy; Interfraction reproducibility; cone-beam computed tomography; 4D-computed tomography.

Conflict of interest: None declared. 


\section{Abstract}

Purpose: To test a prone position against the international-standard supine position in terms of i) feasibility, ii) set-up errors, and iii) respiratory motion in women undergoing whole-breastradiotherapy (WBRT) after wide-local-excision (WLE) of early breast cancer (BC).

Methods: Following WLE of BC with insertion of tumour-bed clips, patients underwent 4D-CT for WBRT-planning in supine and prone positions. Patients were randomised to undergo WBRT fractions 1-7 in either position, switching to the alternate position for fractions 8-15 (40Gy/15fractions total). Cone-beam CT-images (CBCT) were acquired prior to fractions 1, 4, 7, 8, 11 and 14. CBCT data was matched to planning-CT data using a)chest-wall and b)clips. Systematic and random errors were calculated. Maximal displacement of chest-wall and clips with respiration was measured on 4D-CT. Clinical- to planning-target-volume (CTV-PTV) margins were calculated. Patient-comfort-scores and treatment times were compared.

Results: 25 patients were randomized. 192/192 (100\%) planned supine fractions and 173/192 (90\%) prone fractions were completed. Population systematic errors in 3-D ranged from 1.31.9mm (supine) and 3.1-4.3mm (prone) and random errors from 2.6-3.2mm (supine) and 3.8$5.4 \mathrm{~mm}$ (prone) $(\mathrm{p}=0.02)$. Prone positioning reduced chest-wall $(p<0.001)$ and clip motion $(0.5 \pm 0.2 \mathrm{~mm}$ (prone) versus $2.7 \pm 0.5 \mathrm{~mm}$ (supine) $(\mathrm{p}<0.001))$ with respiration. Calculated CTV-PTV margins were greater for prone $(\leq 16 \mathrm{~mm})$ than supine treatment $(\leq 10 \mathrm{~mm})$. Patient-comfortscores and treatment times were comparable $(p=0.06)$.

Conclusions: Set-up errors are significantly worse for prone than supine positioning resulting in larger CTV-PTV margins. Further optimization of the prone treatment platform and technique are required in order to reduce set-up errors before the prone technique can become a viable standard treatment option. 


\section{Introduction}

Standard supine whole-breast radiotherapy (WBRT) is associated with a $1 \%$ non-breast-cancerrelated mortality at 15 years (1) the majority of which is cardiovascular in origin (2). Our data [ref] confirms that prone positioning can reduce heart and left-anterior-descending coronary-artery (LAD) doses in left-breast-affected women of larger cup-size, and lung and chest-wall doses in all women undergoing WBRT. Prone positioning can also reduce normal-tissue doses in some women undergoing partial-breast irradiation (PBI). In order to develop the prone technique into a standard treatment option for these patient populations, further data on its reproducibility (including set-up errors and respiratory motion) are required.

Data suggest that prone treatment is feasible (3-9), but results of studies using electronic-portal imaging (EPI) to investigate inter-fractional variations in breast-tissue position during prone treatment are conflicting $(8,9)$. Morrow et al (9) reported individual-patient systematic errors of $0.1-16.5 \mathrm{~mm}$ with a pooled random error of $5.4 \mathrm{~mm}$ and calculated, using the van Herk formula (10), a CTV-PTV margin of $20 \mathrm{~mm}$ for prone PBI. However, limitations of the study included potential overestimation of set-up errors through use of a patient contour-based match sensitive to soft-tissue distortions, recruitment of 15 patients over a 13-month period, and use of two treatment machines and multiple teams of therapists. More recently, set-up data has been published on 41 patients randomized to supine versus prone treatment (11). Each patient underwent a pre-RT CT-simulator plan-check, and EPI (3 times per week) on treatment. Overall, it was necessary to correct isocentre location or table position in $20 \%$ of supine (higher than expected in an average breast practice) and $20 \%$ of prone patients. The mean length of the displacement vector was greater for prone $(8.1 \pm 4.7 \mathrm{~mm}$ (range $3.0-22.6 \mathrm{~mm})$ ) than supine positioning $(6.6 \pm 3.1 \mathrm{~mm}$ (range $3.0-21.2 \mathrm{~mm})$ ) $(\mathrm{p}=0.02)$. However, supine and prone population systematic errors $(0.8 \mathrm{~mm}$ versus $0.9 \mathrm{~mm})$, and random errors $(3.0 \mathrm{~mm}$ versus $3.9 \mathrm{~mm})$ were comparable $(p=0.06)$. Further randomized data on set-up errors would be of value. In particular, a study using patients as their own controls would be helpful as differences between populations of women treated supine and prone can obscure differences between techniques. As most EPI- 
based verification regimens only detect errors in superior-inferior and anterior-posterior planes and, as increasingly conformal PBI and breast-boost plans are sensitive to left-right errors, conebeam CT (which provides data on translational errors in 3-D) is a useful tool in the ongoing evaluation of set-up errors in the prone position.

In terms of respiratory motion, one small study using four-dimensional (4D)-CT has shown that respiratory-associated movement of the chest wall is significantly reduced in the prone position $(0.1 \pm 0.4 \mathrm{~mm}$ (prone) versus $2.3 \pm 0.9 \mathrm{~mm}$ (supine)) (9). Intra-fraction motion of partial-breast target-volumes is yet to be compared.

A randomized-controlled trial was designed to test a prone position against the international standard supine position in terms of i) feasibility (including patient-comfort, radiographersatisfaction and treatment times), ii) set-up errors, and iii) respiratory motion. Although wholebreast tissue was treated, both chest-wall and clip-based matches were performed such that CTV-PTV margins could be calculated for both WBRT and PBI. 


\section{Methods}

This study was approved by the Royal Marsden Clinical Research and Regional Ethics Committees. All women had undergone WLE for unifocal T1-3 G1-3 invasive-ductal carcinoma or high-grade ductal-carcinoma-in-situ, at which time titanium-clips were inserted into the tumour-bed according to UK protocol (12). Only patients of breast cup-size $\geq C$ (UK) were recruited given our previous dosimetric findings [ref]. Patients requiring nodal irradiation or who had undergone previous breast RT were ineligible.

\section{Patient positioning and 4D-CT imaging}

Patients were positioned on a supine breast board with arms extended above the head in supports (Civco Medical Solutions, lowa, USA). Markers were placed bi-laterally approximately $1-2 \mathrm{~cm}$ posterior to the mid-axillary line, and aligned axially with a midline marker using the lateral laser. Whole-breast tissue was palpated and marked with radio-opaque wire. A coiled belt was placed under tension around lower ribs and epigastrium to record respiratory phase. 4D-CT data (Philips Medical Systems, UK) was acquired without contrast, under free-breathing conditions (3mm slices, C6 to below diaphragm).

Patients were repositioned prone using an in-house-designed prone breast platform comprising an adjustable arm-positioning device registrable to the couchtop, together with a styrofoam/ memory foam mattress [figure 1]. Index breast-tissue hung freely through an aperture in the mattress. Arms were extended comfortably, and the head turned to the contralateral side. Contralateral breast-tissue was pulled laterally. The 4D-CT belt was re-placed around lower ribs and back to record respiratory phase. Bi-lateral tattoos were lined up axially and any anteriorposterior separation recorded. With the CT-simulator couchtop in the zero position in a right-left plane, a fourth marker was placed on the patient's back at the junction of the lateral and longitudinal lasers. Photographs were taken of head, arm, ipsilateral-breast and contralateral breast positions. A prone 4D-CT scan was acquired under free-breathing conditions. Each marker was replaced with a tattoo. 
The time taken to complete each of the supine and prone RT-planning sessions was measured from the time the patient mounted the CT-couch to the time they dismounted. After both scans had been completed, a patient-comfort questionnaire was completed (13). Where a less than maximal comfort-score was reported, patients were asked to report the site(s) of discomfort.

\section{RT planning}

CT data was reconstructed using Philips' Medical Systems software into 10 breathing phases, each defining one-tenth of the breathing-cycle. A free-breathing dataset was also reconstructed and exported to the Pinnacle RT-planning system. Definition of target volumes and organs-atrisk has been described previously [ref]. Standard opposing tangential-fields were employed such that $\geq 90 \%$ of the whole-breast-clinical-target volume (WB-CTV) was encompassed by the $95 \%$ isodose. A total dose of $40 \mathrm{~Gy} / 15$ daily fractions/ 3 weeks/ 6-10MV photons was prescribed to the $100 \%$ isodose.

\section{Radiotherapy treatment and verification}

Patients were randomized into the treatment study only if their prone heart, LAD and lung dosimetry was no worse than that of the supine RT plan. Patients were randomized to start RT in either the prone or the supine position. After fraction 7, patients were switched to the alternate position for the remaining eight fractions.

Each of the supine and prone positions was reproduced at treatment by aligning tattoos to lasers, using photographs for assistance where necessary. For each position, visual checks were made of the relationship of field edges to tattoos and breast tissue. Where this relationship was out by more than $10 \mathrm{~mm}$, the patient was set-up again. Once set-up was satisfactory, set-up errors were measured for clinical purposes using EPI (iView, Elekta, Crawley, UK) acquired during treatment delivery on fractions 1-3 and 8-10. A shift was applied if the result was $>5 \mathrm{~mm}$ in any of the cardinal directions on at least two consecutive days. Where errors of $>10 \mathrm{~mm}$ occurred in different directions on consecutive days, treatment was stopped and patients completed their RT course in the supine position. 
On-treatment cone-beam kV-CT (CBCT) images of the chest were acquired immediately after set-up at fractions 1, 4, 7, 8, 11 and 14 using the Elekta Synergy X-Ray Volume Imaging system (Elekta, Stockholm, Sweden). Approximately 350 projections were collected over a $180^{\circ}$ rotation of the gantry (known to provide clear resolution of chest-wall, heart and lungs (14) and tested on a phantom pre-study to confirm that titanium-clips would be visible). Exposure settings were $100 \mathrm{kV}$ and $350 \mathrm{mAs}$. Image acquisition took $\sim 30$ seconds and the technique resulted in an ipsilateral breast dose of $\sim 17 \mathrm{mGy}$ per scan (measured using a phantom and consistent with previous data (15)). Projections were reconstructed into a $200 * 200 * 128 \mathrm{~mm}$ volume at a $2 \mathrm{~mm}^{3}$ voxel resolution. Where an isocentre-shift had been recommended on the basis of EPI, CBCTimaging was acquired prior to the shift. All CBCT data was analysed off-line. The CBCT volume was manually registered to the reference planning-CT using a) a chest wall/ sternum match, and b) a titanium-clip-based match. The correction reference point was set to the isocentre.

Fractions 1, 7, 8 and 14 were timed from the patient mounting the treatment couch to the patient dismounting. After each fraction, patient-comfort and radiographer-satisfaction questionnaires (13) were completed. Patients were asked to comment on any sources of discomfort and radiographers on any sources of dissatisfaction.

\section{Analysis}

CBCT registration results were analyzed for each patient. Mean displacements and standard deviations (SD) of chest-wall and clips were calculated in 3-dimensions for each patient in each position. Population systematic errors $(\Sigma)$ were calculated, for chest-wall and clips, from the SD of all means, and population random errors $(\sigma)$ from the root mean square (RMS) of all SD (16). Results were compared using the paired t-test. CTV-PTV margins were calculated based on the van-Herk formula $(2.5 \Sigma+0.7 \sigma(10))$ with linear addition of errors due to respiratory motion.

In order to assess chest-wall motion with respiration, two axial slices were reviewed consistent with a previous study (9), one in the plane of the nipple (typically T6-7 level for supine datasets and T9-10 level for prone datasets), and one approximately half-way through T4. Maximal chestwall displacement in a plane perpendicular to the couch-top was calculated. In order to assess 
the effect of respiratory motion upon tumour-bed (TB), the maximal displacement of the most mobile titanium-clip was measured in each of the anterior-posterior (perpendicular to couch-top), superior-inferior (vertical) and left-right planes. Mean displacements for prone and supine positions were compared using the paired t-test.

Patient comfort and acceptability was summarized from the questionnaire in a patient-comfort score ranging from 0 (least satisfactory) to 9 (most satisfactory). Radiographer satisfaction with positioning technique was summarized on a satisfaction scale measured using a linear analogue score ranging from 0 (most satisfactory) to 12 (least satisfactory). Median scores, simulation and treatment times were compared using Wilcoxon signed-rank tests. 


\section{Results}

Twenty-five patients were randomized. Median age was 56 years (range 41-75). Median patientreported cup-size was D (range C-G) (equivalent to WB-CTV $1053 \mathrm{~cm}^{3}$ (range 394-2051)).

Numbers of patients completing each part of the treatment, and changes required to treatmentplans are shown in table 1. In 3/4 patients who did not complete prone RT, set-up errors were out-of-tolerance. The fourth patient had to be re-positioned three times over 45 minutes before set-up was clinically satisfactory. The first EPI was then out-of-tolerance and, as the patient was in severe discomfort by this stage, it was decided to complete treatment in the supine position.

Individual patient systematic and random errors in each of the cardinal directions based on a chest-wall match are reported in figure 2. Previous work has demonstrated that set-up errors improve with time (8). However, mean displacements in the superior-inferior direction worsened in the middle of our study. Two prone platforms were in use (one in planning and one on treatment). The top layer of each was constructed of memory foam, and it was suspected that the treatment foam, being in more regular use, may have been compressed to a greater extent than the planning foam. From patient 18 onwards, patients were treated using the foam on which they had been planned. Superior-inferior errors were lower in subsequent patients.

The magnitude of individual patient set-up errors did not correlate with WB-CTV, chest or epigastric circumference, age or radiographer-satisfaction score. Difficulties with shoulder positioning were mentioned by radiographers in association with 4/5 patients whose set-up errors exceeded $10 \mathrm{~mm}$. Underarm discomfort and/or shoulder tension was found in 4/4 patients who failed to complete prone RT. Placement of contralateral tattoo in a skin fold, dorsal tattoo $>5 \mathrm{~cm}$ from midline, epigastric circumference $>40 \mathrm{~cm}$, and initial seroma volume $>25 \mathrm{~cm}^{3}$ each contributed to set-up problems in 2/4 patients.

Population mean displacements, systematic and random errors based on each of chest-wall and clip matches are listed in table 2. Prone positioning reduced chest-wall and clip motion with respiration, most markedly in the anterior-posterior plane [table 3]. Table 4 shows CTV-PTV margins calculated using both set-up and respiratory motion errors. 
Median patient-comfort scores (PCS) at planning were 9 (range 6-9) (supine) versus 8 (5-9) (prone) $(\mathrm{p}=0.06)$, and at treatment were 8.5 (range 5-9) (supine) and 8 (range 3-9) (prone) $(p=0.07)$. In the prone position, more women reported neck $(4 / 25$ versus $0 / 25, p=0.01)$ and inframammary discomfort $(6 / 25$ versus $0 / 25, p=0.001)$ than in the supine position. Median radiographer-satisfaction scores were 0 (range $0-5)$ (supine) and 1 (range 0-10) (prone) $(\mathrm{p}=0.04)$. The most frequent cause of dissatisfaction was difficulty in aligning tattoos. Many radiographers commented on the variability of lateral and dorsal tattoo position with arm and shoulder position despite use of the arm-positioning device. Some patients had difficulty in maintaining their grip on the T-bar handles whilst radiographers shifted the torso in order to align tattoos. In particular, in patients with lateralized dorsal tattoos, shoulder position (via effects upon scapula position) caused considerable variability in the position of the dorsal tattoo such that it was difficult to align tattoos in an axial plane.

Mean times taken to complete planning scans were 15 (range 12-25) minutes (supine) and 20 (range 12-30) minutes (prone) $(p=0.03)$. Longest planning times for the prone position were 30 minutes (first three patients). From patient 4 onwards, no patients took more than 25 minutes.

Mean treatment times were 20(10-34)minutes (supine) and 21(10-40)minutes (prone) $(\mathrm{p}=0.3)$. Figure 4 demonstrates that the majority of women were set-up, cone-beam imaged and treated in $\leq 20$ minutes for both positions. 


\section{Discussion}

This randomized-controlled study aimed to test a prone position against the international standard supine position in terms of i) feasibility, ii) patient positional errors, and iii) respiratory motion in women undergoing WBRT after WLE of early BC. 21/25 (84\%) patients satisfactorily completed $173 / 192(90 \%)$ planned prone RT fractions. This is fewer than reported in previous feasibility studies of the prone position (3-9). This may have been because our protocol specified tolerance criteria for set-up errors beyond which women were required to complete treatment in a supine position. 3/4 women who did not complete treatment had set-up errors which exceeded tolerance criteria. In the remaining patient, set-up was prolonged and uncomfortable. It is possible that, without the availability of a supine plan, the patient and radiographers might have persisted with prone treatment but, in practice, prone RT was unfeasible due mainly to suboptimal tattoo placement.

The ranges of individual prone systematic and random set-up errors are similar to those reported previously $(9,11)$. Our population systematic and random errors are similar to one previous study (9) but worse than reported in another (11). Both chest-wall and clip-based matches were associated with larger set-up errors for prone as compared to supine positioning, particularly in a superior-inferior plane. Contrary to previous reports, which suggest that set-up errors for partialbreast target volumes are worse than for whole-breast volumes due to TB shrinkage over time (17), our calculated margins were similar for both chest-wall and clip-based matches. This reflects the practice of full-thickness excision-cavity closure at our institution which results in small TB volumes (containing low-volume seroma) that are relatively stable with time.

Factors which may have contributed to larger prone set-up errors or failure to complete prone RT include platform design, set-up technique, study design and patient factors. Platform design was based on that used by the team with the greatest experience in prone breast RT (New York University Hospital). A simple Styrofoam and memory foam platform has been reported to be comfortable and portable (7), the use of an arm-positioning device aiding reproducibility of arm and shoulder position as well as indexing the platform to the couch. However, pre-CBCT set-up 
errors using such a device have not yet been reported. Most of our patients found the prone position to be reasonably comfortable. Indeed, the only patient whose discomfort contributed to failure of the prone position had been on the platform for 45 minutes. However, radiographers frequently raised concerns over arms and shoulder positioning. Despite the stable T-bar position, several patients were able to position their shoulders and arms quite differently whilst satisfactorily gripping the T-bar and setting up to in-room lasers. Close attention had to be paid to photographs in order to try to reproduce upper limb position in such patients. As photographs are less accurate than tattoos, poor reproducibility of upper limb position is likely to have contributed significantly to set-up errors (particularly those in anterior-posterior and superiorinferior planes (because patients brace themselves from relaxing into the ipsilateral hole)). It may be that immobilization of shoulders and upper arms, for example using Vacu-bags, would have reduced errors.

Tattoo position could have been improved. Our study design required that patients be treatable in both supine and prone positions, contributing to difficulties in selecting optimal tattoo position. In order to minimize the number of tattoos, lateral tattoos were placed as posteriorly as possible in the supine position such that they would be unobscured by contralateral breast-tissue in the prone position. This was achieved in most patients but in $2 / 4$ patients who failed to complete prone treatment, the contralateral tattoo was in a skin fold and therefore unusable for prone positioning. In the prone position it was also noted that shoulder and arm position had a significant effect upon the superior-inferior position of tattoos. More inferior placement of tattoos may have lead to more stability in their position and therefore to reduced set-up errors. It may be that the prone position cannot be reliably reproduced without increasing the number of tattoos. NYUH make use of an additional tattoo on the lateral aspect of the affected breast but this might not be cosmetically acceptable to all patients. Alternatively, the position of the patient's torso on the prone platform might be better reproduced using a second dorsal tattoo placed more inferiorly allowing the torso to be straightened. 
Another study-design feature that might have contributed to set-up errors was use of the belt to monitor respiration (present at 4D-CT but not during treatment). In some patients, this belt lifted epigastric-tissue out of the mattress aperture thereby pulling breast-tissue inferiorly at planning. Without the belt in-situ, breast-tissue may have hung more freely contributing to superior-inferior/ anterior-posterior set-up errors.

Previous work has suggested that involvement of different teams of radiographers over time contributes to set-up errors (9). The 25 patients in our study were recruited, planned and treated within an eight-month time frame, predominantly using one treatment machine and one team of radiographers (six personnel). There was insufficient difference in the experience-level of different radiographers to be able to relate set-up errors to experience. In only one case did suboptimal tattoo placement occur in association with an inexperienced radiographer joining the planning team.

Patient factors that might have contributed to failure of the prone position include body habitus (because increased subcutaneous fat leads to more mobile tattoos) and, in a couple of patients, tumour bed seroma (larger seromas shrink with time leading to displacement of clips). However, the impact of such factors has been minimized by using patient as their own controls.

Other criticisms of study methodology include non-automated matching of clips. In most patients it was possible manually to match clips on planning and cone-beam images but in a few patients, the clips could not be lined up completely, due to breast-tissue distortion and/or a change in the TB itself between planning and treatment. This meant that in some cases there were errors of $1-2 \mathrm{~mm}$ in the clip-matching process. Fully-automated clip matching (18) would have been more robust and would be crucial to reducing image-processing times were CBCT to become part of standard verification protocols.

Our 4D-CT analysis confirms that prone positioning reduces anterior chest-wall motion with respiration by a similar extent to that reported previously (9), but also demonstrates reduced clipmotion with respiration, particularly in the anterior-posterior direction. The reduction of respiratory motion compensates in part for the larger set-up errors in the prone position but 
overall this study finds the CTV-PTV margins required for prone treatment to be significantly greater than those required for supine treatment. Adding respiratory motion to set-up errors generated WBRT CTV-PTV margins of $10 \mathrm{~mm}$ for supine treatment and $12-16 \mathrm{~mm}$ for prone treatment, and PBI CTV-PTV margins of $6-9 \mathrm{~mm}$ for supine and $13-15 \mathrm{~mm}$ for prone treatment. Clinical implications of these margins have not been determined in this patient cohort but previous work (19) has found for PBI that, for every $10 \mathrm{~mm}$ increase in CTV-PTV margins, the mean heart dose doubles (from 23-43cGy) and mean lung dose triples (from 61 to 180cGy). Therefore, CTV-PTV margins of these magnitudes could negate the dosimetric benefits of prone positioning in the context of $\mathrm{PBI}$.

What is the future of prone treatment? The dosimetric benefits and patient populations to which these apply are now well-documented. Prone treatment appears to be feasible and we found it to be well-tolerated by patients, acceptable to radiographers and comparable to supine WBRT in terms of planning/treatment times in most women. These findings are encouraging in the context of a novel technique being tested for the first time in a busy department. However, set-up errors have now been reported to be significantly greater for prone versus supine positioning in $2 / 3$ studies including ours. Set-up errors are likely to be reduced by optimizing platform design and tattoo placement, and by increasing experience within a team. The largest experiences are being amassed by teams in New York and in Hungary and it is encouraging that these departments continue to refine their respective techniques in order to be able to offer the dosimetric benefits of prone positioning to appropriate women. Another means by which prone treatment could become a viable standard treatment option is to optimize verification. CBCT as used here and in NYUH is one possibility but, given the potential long-term risks of exposure to low-dose ionizing radiation $(20,21)$, may not be justifiable in the context of patients with low-risk disease, especially given that the basis for pursuing prone treatment is reduction of long-term side-effects. Other technologies that can be used to localize soft tissue targets include implanted transponder systems, implantable dosimeters, and opto-electronic surface imaging (22-24). 
Collaboration between centres investigating prone breast RT and industry will be helpful to its development as a standard treatment option for larger-breasted women worldwide.

In conclusion, we have found the prone technique to be feasible, but systematic and random set-up errors to be significantly worse than for the supine technique. Further optimization of the treatment platform, technique, and verification strategies will be required before this technique can become a viable standard treatment option. 


\section{References}

1. Clarke M, Collins R, Darby $\mathrm{S}$, et al. Effects of radiotherapy and of differences in the extent of surgery for early breast cancer on local recurrence and 15-year survival: an overview of the randomised trials. Lancet 2005;366:2087-2106.

2. Darby SC, McGale P, Taylor CW, et al. Long-term mortality from heart disease and lung cancer after radiotherapy for early breast cancer: prospective cohort study of about 300,000 women in US SEER cancer registries. Lancet Oncol 2005;6:557-565.

3. Grann A, McCormick B, Chabner ES, et al. Prone breast radiotherapy in early-stage breast cancer: a preliminary analysis. Int J Radiat Oncol Biol Phys 2000;47:319-325.

4. Mahe MA, Classe JM, Dravet F, et al. Preliminary results for prone-position breast irradiation. Int J Radiat Oncol Biol Phys 2002;52:156-160.

5. Goodman KA, Hong L, Wagman R, et al. Dosimetric analysis of a simplified intensity modulation technique for prone breast radiotherapy. Int $J$ Radiat Oncol Biol Phys 2004;60:95-102.

6. McKinnon R, Christie D, Peres $\mathrm{H}$, et al. The prone technique for breast irradiation - is it ready for clinical trials? Breast 2009;18:30-34.

7. Formenti SC, Gidea-Addeo D, Goldberg JD, et al. Phase I-II trial of prone accelerated intensity modulated radiation therapy to the breast to optimally spare normal tissue. $J$ Clin Oncol 2007;25:2236-2242.

8. Varga Z, Hideghety K, Mezo T, et al. Individual Positioning: A Comparative Study of Adjuvant Breast Radiotherapy in the Prone Versus Supine Position. Int J Radiat Oncol Biol Phys 2009.

9. Morrow NV, Stepaniak C, White J, et al. Intra- and interfractional variations for prone breast irradiation: an indication for image-guided radiotherapy. Int $J$ Radiat Oncol Biol Phys 2007;69:910-917. 
10. van Herk M, Remeijer P, Rasch $C$, et al. The probability of correct target dosage: dosepopulation histograms for deriving treatment margins in radiotherapy. Int $\mathrm{J}$ Radiat Oncol Biol Phys 2000;47:1121-1135.

11. Varga Z, Hideghety $\mathrm{K}$, Mezo $\mathrm{T}$, et al. Individual positioning: a comparative study of adjuvant breast radiotherapy in the prone versus supine position. Int $J$ Radiat Oncol Biol Phys 2009;75:94-100.

12. Coles $\mathrm{CE}$, Wilson CB, Cumming J, et al. Titanium clip placement to allow accurate tumour bed localisation following breast conserving surgery: audit on behalf of the IMPORT Trial Management Group. Eur J Surg Oncol 2009;35:578-582.

13. Nutting CM, Khoo VS, Walker V, et al. A randomized study of the use of a customized immobilization system in the treatment of prostate cancer with conformal radiotherapy. Radiother Oncol 2000;54:1-9.

14. McBain CA, Henry AM, Sykes J, et al. X-ray volumetric imaging in image-guided radiotherapy: the new standard in on-treatment imaging. Int $J$ Radiat Oncol Biol Phys 2006;64:625-634.

15. Islam MK, Purdie TG, Norrlinger BD, et al. Patient dose from kilovoltage cone beam computed tomography imaging in radiation therapy. Med Phys 2006;33:1573-1582.

16. van Herk M. Errors and margins in radiotherapy. Semin Radiat Oncol 2004;14:52-64.

17. Hasan Y, Kim L, Martinez A, et al. Image Guidance in External Beam Accelerated Partial Breast Irradiation: Comparison of Surrogates for the Lumpectomy Cavity. Int $J$ Radiat Oncol Biol Phys 2007;70:619-625.

18. Kim LH, Wong J, Yan D. On-line localization of the lumpectomy cavity using surgical clips. Int J Radiat Oncol Biol Phys 2007;69:1305-1309.

19. Cox BW, Horst KC, Thornton S, et al. Impact of increasing margin around the lumpectomy cavity to define the planning target volume for 3D conformal external beam accelerated partial breast irradiation. Med Dosim 2007;32:254-262. 
20. Hall EJ, Wuu CS. Radiation-induced second cancers: the impact of 3D-CRT and IMRT. Int J Radiat Oncol Biol Phys 2003;56:83-88.

21. Frankenberg D, Kelnhofer $\mathrm{K}$, Bar $\mathrm{K}$, et al. Enhanced neoplastic transformation by mammography $X$ rays relative to $200 \mathrm{kVp} X$ rays: indication for a strong dependence on photon energy of the RBE(M) for various end points. Radiat Res 2002;157:99-105.

22. Bert C, Metheany KG, Doppke KP, et al. Clinical experience with a 3D surface patient setup system for alignment of partial-breast irradiation patients. Int $J$ Radiat Oncol Biol Phys 2006;64:1265-1274.

23. Scarantino CW, Rini CJ, Aquino M, et al. Initial clinical results of an in vivo dosimeter during external beam radiation therapy. Int J Radiat Oncol Biol Phys 2005;62:606-613.

24. Willoughby TR, Kupelian PA, Pouliot $J$, et al. Target localization and real-time tracking using the Calypso 4D localization system in patients with localized prostate cancer. Int $J$ Radiat Oncol Biol Phys 2006;65:528-534. 


\begin{tabular}{|c|c|c|c|c|c|}
\hline $\begin{array}{l}\text { Treatment } \\
\text { position }\end{array}$ & $\begin{array}{l}\text { No. of patients } \\
\text { completing } \\
\text { planned number } \\
\text { of fractions }\end{array}$ & $\begin{array}{l}\text { No. of } \\
\text { planned } \\
\text { fractions } \\
\text { completed }\end{array}$ & $\begin{array}{l}\text { No. of patients } \\
\text { requiring change } \\
\text { to planned } \\
\text { treatment }\end{array}$ & $\begin{array}{l}\text { Isocentre } \\
\text { shifts }\end{array}$ & $\begin{array}{l}\text { Increases to } \\
\text { field borders }\end{array}$ \\
\hline Supine & $25(100 \%)$ & $\begin{array}{l}192 / 192 \\
(100 \%)\end{array}$ & $2 / 25$ & $5 \mathrm{~mm}$ ant & $5 \mathrm{~mm}$ ant \\
\hline Prone & 21 (84\%) & $\begin{array}{l}173 / 192 \\
(90 \%)\end{array}$ & $8 / 25$ & $\begin{array}{l}5 \mathrm{~mm} \text { posterior } \\
8 \mathrm{~mm} \text { inferior } \\
8 \mathrm{~mm} \text { anterior } \\
5 \mathrm{~mm} \text { inferior } \\
8 \mathrm{~mm} \text { anterior } \\
5 \mathrm{~mm} \text { posterior }\end{array}$ & $\begin{array}{l}5 \mathrm{~mm} \text { inferior } \\
5 \mathrm{~mm} \text { anterior* }\end{array}$ \\
\hline
\end{tabular}

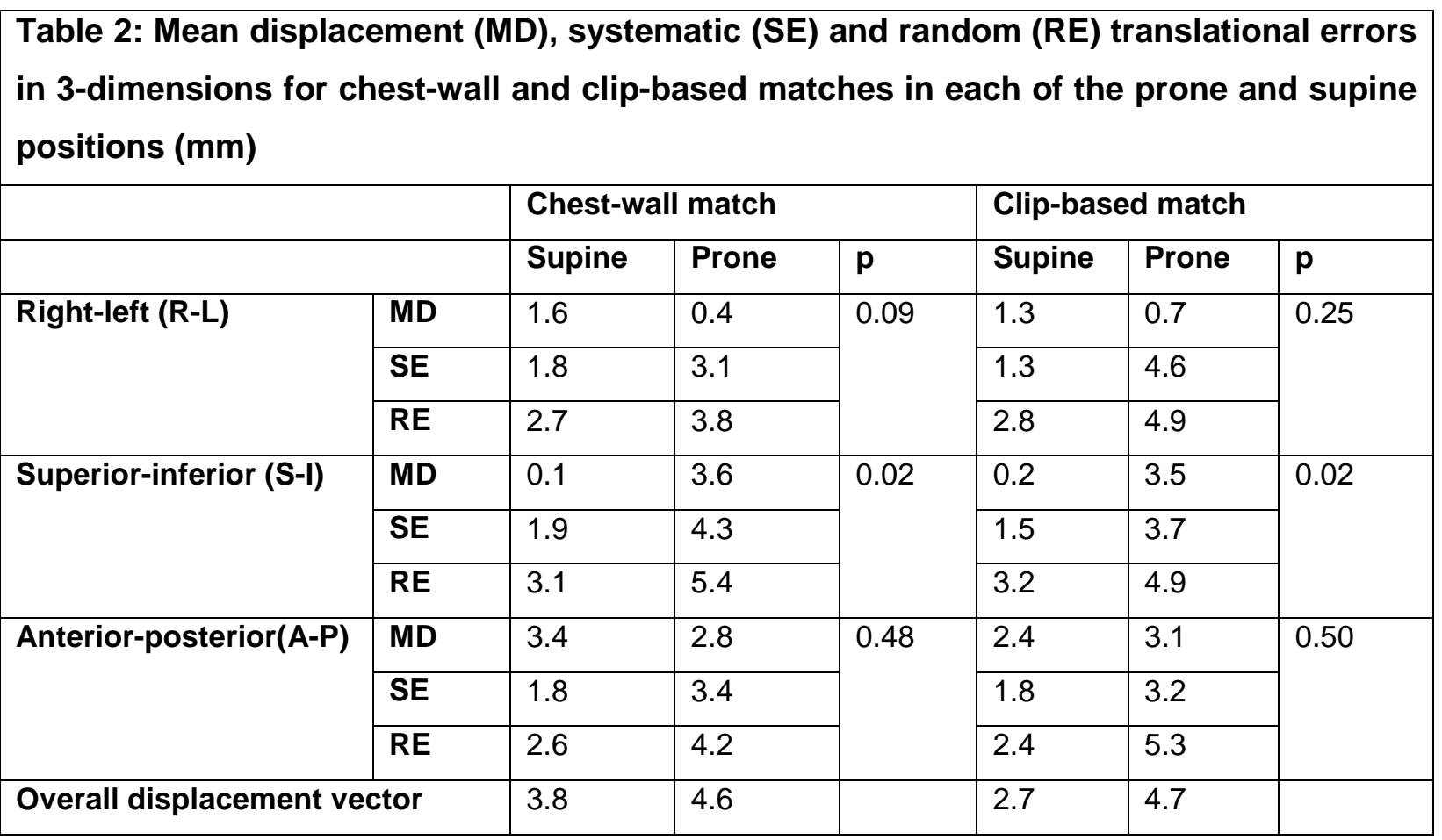




\begin{tabular}{|c|c|c|c|c|}
\hline & & Supine & Prone & $\mathbf{p}$ \\
\hline $\begin{array}{l}\text { Chest-wall displacement (A-P } \\
\text { Mean value } \pm 95 \% \mathrm{Cl} \text { (range) }\end{array}$ & & $3.3 \pm 0.5(1-7)$ & $1.1 \pm 0.3(0-2)$ & $<0.001$ \\
\hline Clip displacement & R-L & $1.1 \pm 0.4(0-3)$ & $0.1 \pm 0.1 \quad(0-1)$ & $<0.001$ \\
\hline Mean value $\pm 95 \% \mathrm{Cl}$ (range) & S-I & $1.0 \pm 0.4(0-3)$ & $0.2 \pm 0.2(0-1)$ & 0.001 \\
\hline & A-P & $2.7 \pm 0.5(0-6)$ & $0.5 \pm 0.2(0-2)$ & $<0.001$ \\
\hline
\end{tabular}

\begin{tabular}{|c|c|c|c|c|}
\hline \multirow[b]{2}{*}{ Margin } & \multicolumn{2}{|c|}{ Chest-wall-based match } & \multicolumn{2}{|c|}{ Clip-based match } \\
\hline & Supine & Prone & Supine & Prone \\
\hline R-L & 9.7 & 11.5 & 6.3 & 12.8 \\
\hline S-I & 10.2 & 15.6 & 7.0 & 12.9 \\
\hline A-P & 9.6 & 12.5 & 8.9 & 14.7 \\
\hline
\end{tabular}


Figure 1: $\quad$ Prone treatment platform (styrofoam base layer with memory foam top layer, mobile longitudinal scale and arm positioning device)

Figure 2: Individual patient a) systematic and b) random translational set-up errors plotted by patient number (in order of treatment)

Figure 3: $\quad$ Population mean displacements for a) chest-wall based match and b) clipbased match

Figure 4: $\quad$ Times taken to complete RT-treatment sessions (including patient set-up, CBCT and treatment) 


\section{Figure 1.}

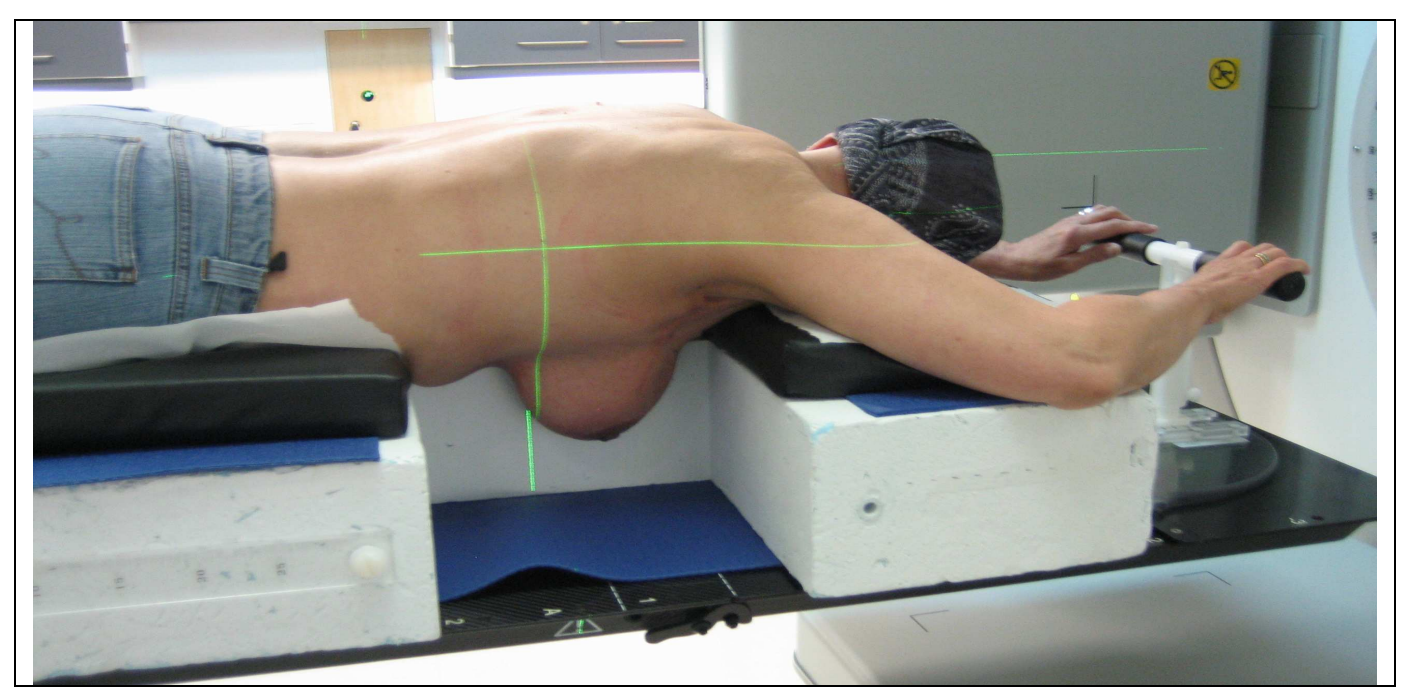




\section{Figure 2.}

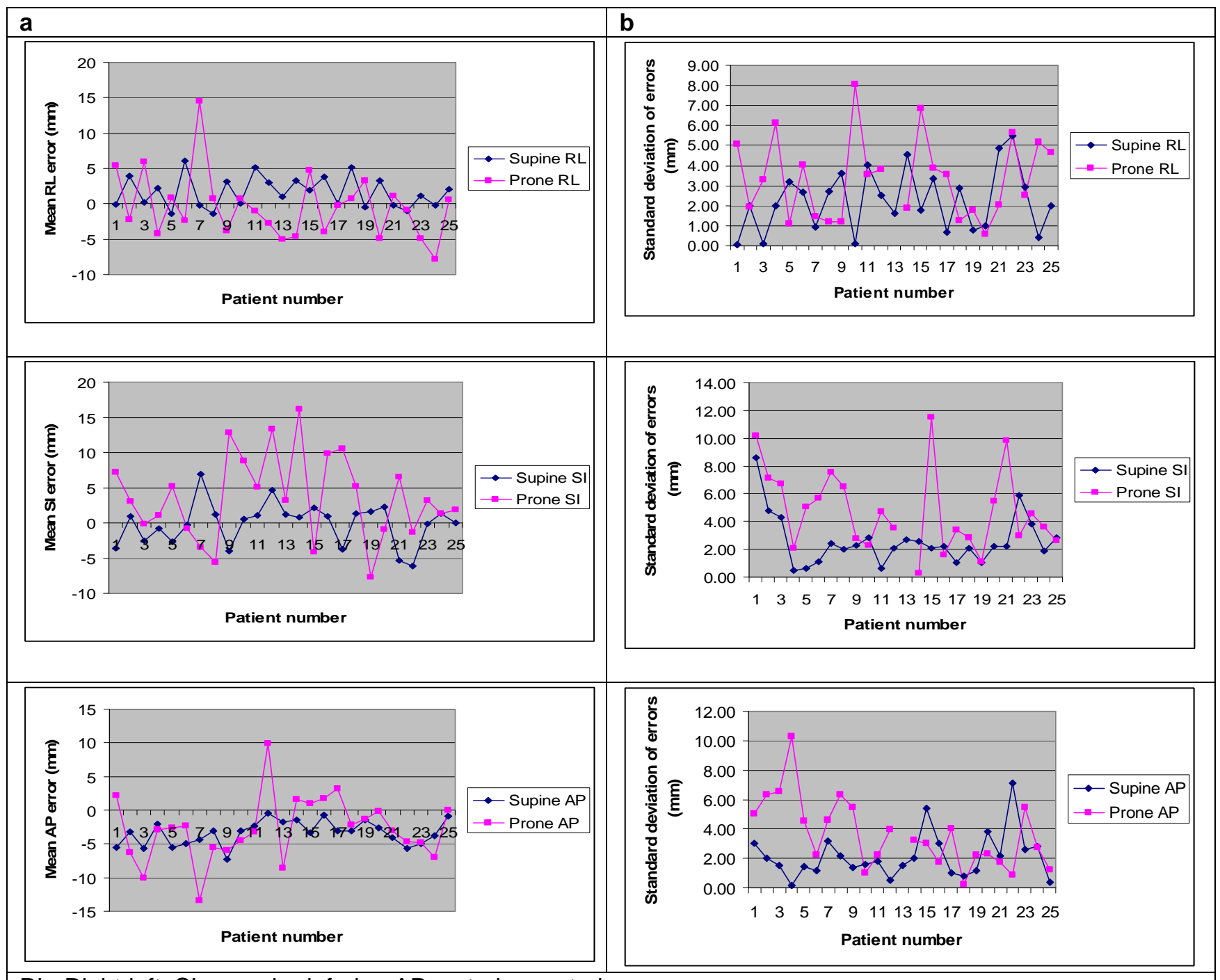

$\mathrm{RL}=$ Right-left; $\mathrm{SI}=$ superior-inferior; $\mathrm{AP}=$ anterior-posterior 
Figure 3.

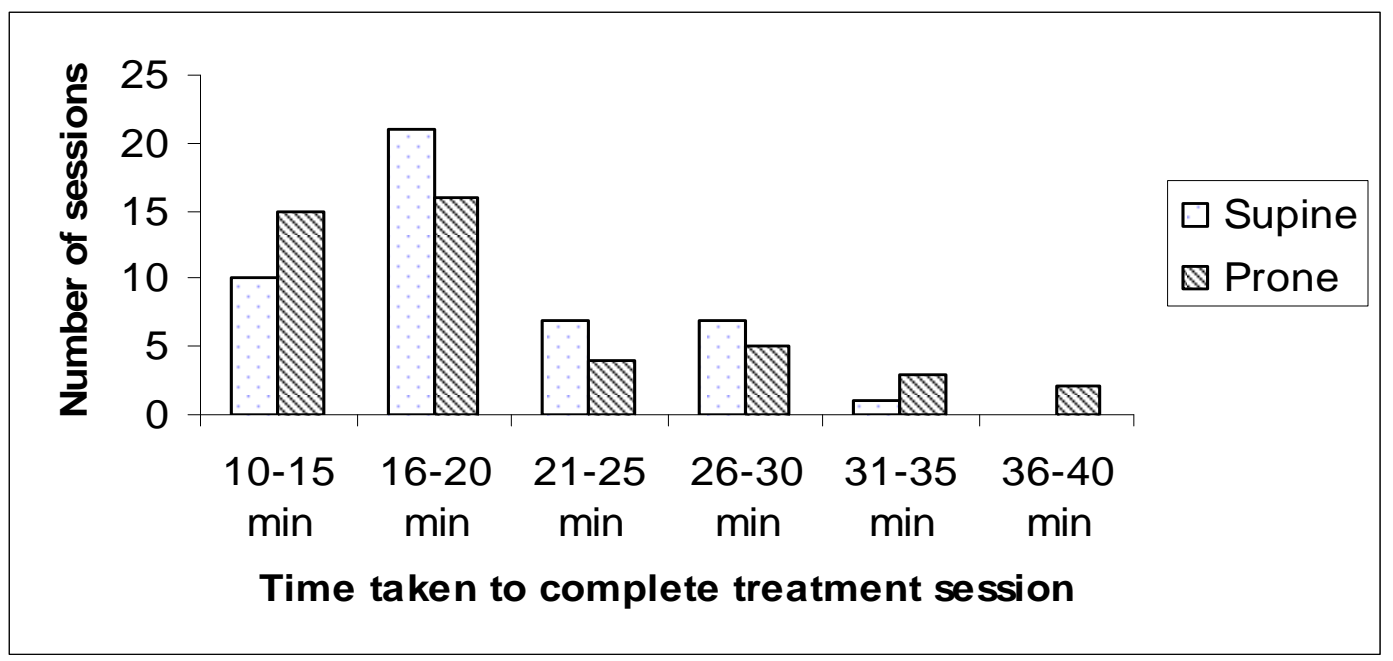

